\title{
RÉFUGIÉS DU SALVADOR ADMIS AU CAN
}

Le ministre canadien de l'Emploi et de l'Immigration, M. Lloyd Axworthy, a annoncé le 19 mars dernier des mesures spéciales pour venir en aide aux personnes qui ont été touchées par le conflit au Salvador.

- Les Salvadoriens qui ont été autorisés à séjourner au Canada et qui y ont des parents capables de les parrainer à titre d'immigrants pourront obtenir le droit de s'établir au Canada en tant que résidents permanents, pourvu qu'ils répondent aux exigences relatives à la santé et à la sécurité.

- Les visiteurs autorisés en provenance du Salvador qui n'ont pas de parents au Canada mais qui désirent s'y établir en permanence obtiendront un permis du Ministre et l'autorisation de chercher un emploi. Le dossier de ces personnes sera réexaminé après six mois. Si l'on constate qu'elles pourront s'établir avec succès au Canada, elles se verront accorder le droit d'établissement, pourvu qu'elies satisfassent aux exigences réglementaires.
- Les Canadiens et les résidents permanents de ce pays dont les parents ont dû fuir le Salvador pourront aider les membres de leur famille à venir au Canada en vertu de critères élargis, pourvu qu'ils soient en mesure de prendre des dispositions satisfaisantes en vue de leur réétablissement.

- Les Salvadoriens en séjour autorisé au Canada, qui ne veulent pas rentrer dans leur pays tant que la situation ne s'y sera pas normalisée pourront s'adresser à un Centre d'Immigration Canada pour obtenir une prolongation de séjour. Ceux qui auront besoin de travailler seront légalement autorisés à le faire.

Monsieur Axworthy a fait observer que ces mesures cadrent avec la nécessité reconnue de suivre de près la situation au Salvador et de continuer a prendre les mesures nécessaires pour faire face à l'aspect humanitaire du problème.

Le Ministre a précisé que le HCNUR estime qu'il sera possible de rapatrier la plupart de ces personnes qui

\section{PROJET DE PROGRAMME SPÉCIAL À L'INTEN]}

Après la visite d'une équipe spéciale en Amérique centrale, le Comité inter-Églises pour les réfugies, le Comité inter-Églises pour les droits de la personne en Amérique latine et le Comité chrétien pour les droits de l'homme en Amérique latine ont présenté au ministère de l'Emploi et de l'Immigration une proposition visant à accentuer les mesures prises pour aider les réfugiés du Salvador. En voici des extraits:

\section{HISTORIQUE}

Le climat de violence et de répression qui sévit en Amérique centrale et qui s'est particulièrement envenimé au cours de la dernière année a obligé cent mille réfugiés à s'enfuir pour aller chercher protection dans les pays avoisinants. Venus surtout du Salvador et, dans une moindre mesure, du Guatemala, ils se trouvent en nombre relativement élevé au Mexique, à Belize, au Honduras et au Costa Rica. Les autres pays de la région en ont également accueilli un petit nombre.

A Belize et au Honduras, la plupart des réfugiés sont d'origine paysanne ou "campesino" et bénéficient de l'aide du HCNUR et d'organismes bénévoles. Tous les intéressés s'entendent pour dire que ces réfugiés ne seraient pas en mesure de se réétablir à l'extérieur de la région. La vie est difficile pour eux, et l'aide d'urgence qui continue de leur être accordée, de même que les petits projets de développement économique axés sur l'autonomie adoptés par la communauté des nations à leur intention représentent autant de moyens appropriés et très nécessaires.
Au Costa Rica et au Mexique, cependant, il en va tout autrement. En effet, on y trouve, parmi les réfugiés, de nombreux travailleurs instruits et spécialisés, des étudiants, des enseignants (qui forment une classe à part) et d'autres qui ont besoin d'aide pour s'établir de façon productive. Au Costa Rica, ces personnes sont considérées comme des réfugiés au sens de la Convention des Nations Unies et, à ce titre, ne peuvent être expulsées. Elles ne sont toutefois pas autorisées à travailler ni à suivre des cours de formation professionnelle; elles sont donc obligées de subsister à même la maigre allocation que leur offrent les Nations Unies ou d'occuper illégalement un emploi, ce qui pourrait compromettre leur statut de réfugié. Avant le 3 mars 1981, lorsque les Nations Unies ont signé un accord avec les autorités du Mexique, la situation dans ce pays était encore pire. Les réfugiés n'étaient pas reconnus comme tels et n'étaient pas autorisés à travailler; ils recevaient quand même une aide limitée des Nations Unies par l'entremise de la Croix Rouge. Heureusement, le nouvel accord réglera le problème de la reconnaissance du statut de réfugié et permettra de régulariser les programmes d'aide d'urgence, mais l'on s'attend généralement à ce que les réfugiés finissent par se retrouver dans la même situation que ceux cantonnés au Costa Rica.

Au Canada, il y a relativement peu de réfugiés d'Amerique centrale. La plupart d'entre eux ont des parents, des membres de leur famille, des amis intimes ou des anciens collègues qui sont actuellement victimes de persécution et qui doivent absolument quitter leur pays. Certains ont des liens avec des gens qui sont déjà au nombre des réfugiés se trouvant au Mexique et 
ont fui le Salvador lorsque les combats y auront pris fin. Le HCNUR avise donc tous les pays d'accueil que, pour le moment, il n'y a pas lieu de mettre sur pied des programmes spéciaux de réétablissement des réfugiés salvadoriens. Nous partageons cet avis, mais certaines personnes ne pourront peut-être pas rentrer dans leur pays parce qu'elles ont des raisons précises de craindre la persécution. C'est pourquoi nous sommes disposés à accepter les demandes que les personnes dans cette situation présentent à titre de réfugiés dans nos bureaux régionaux.

Aux termes de la législation actuelle en matière d'immigration, les groupes privés ont le droit de parrainer des réfugiés de n'importe quelle partie du monde.

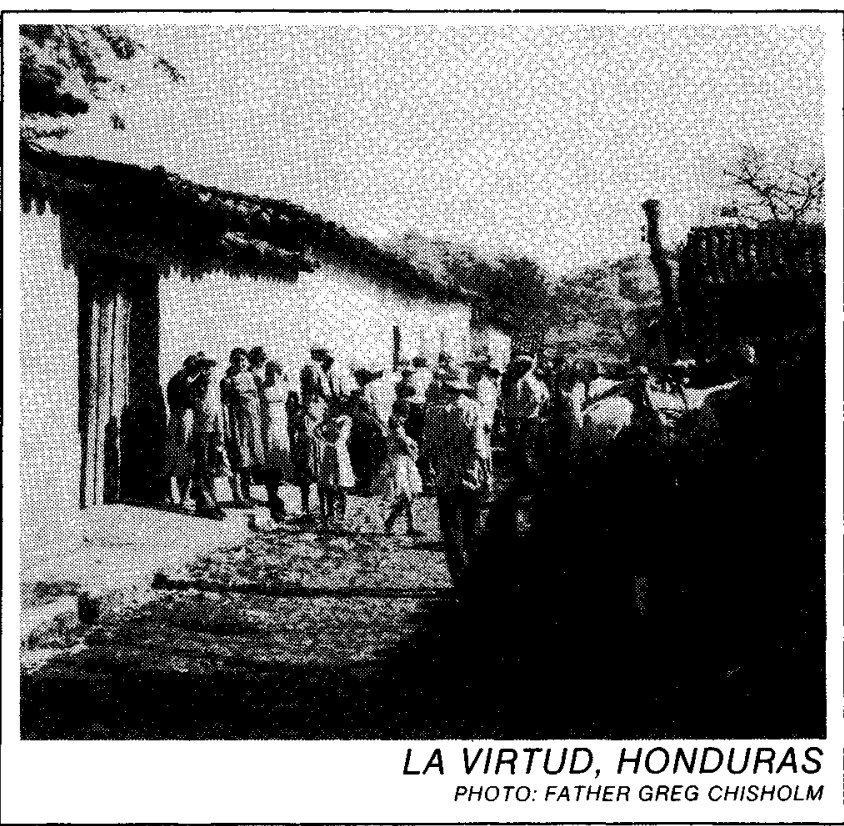

\section{ION DES RÉFUGIÉS D'AMÉRIQUE CENTRALE}

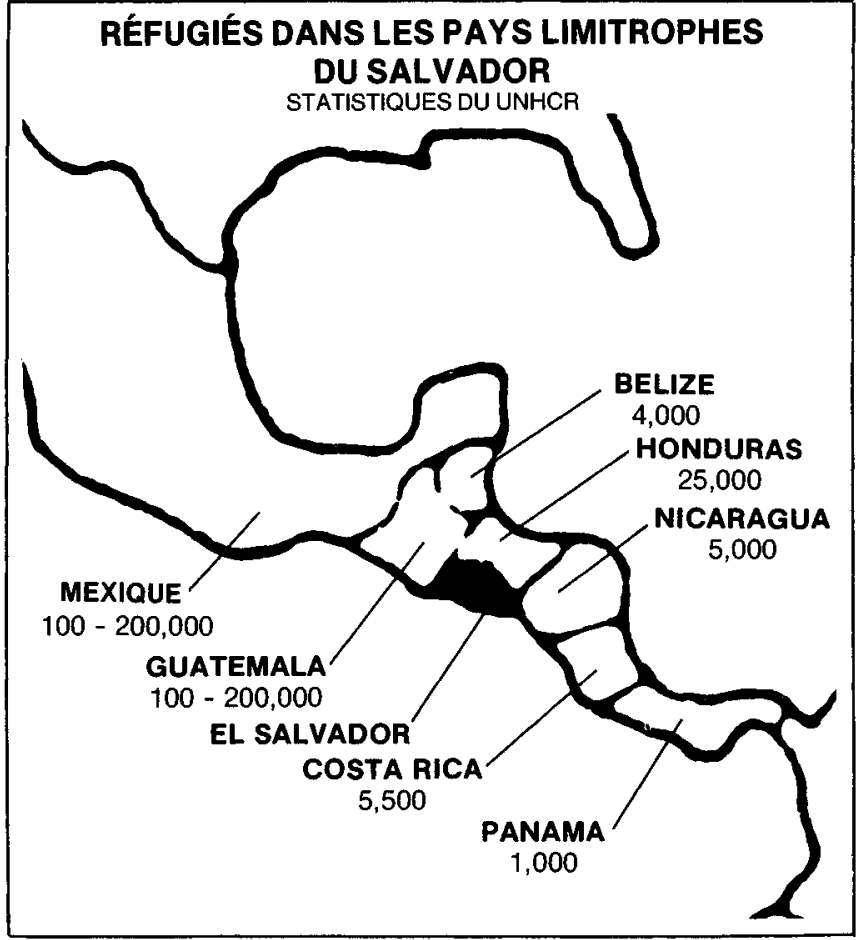

au Costa Rica. Le bureau d'Immigration Canada au Mexique étudie en priorité le cas des personnes qui peuvent être parrainées à titre de "membres de la catégorie de la famille". Bien d'autres cependant ne remplissent pas les conditions voulues pour rentrer dans cette catégorie ou celle des parents aidés, car bien souvent le parent en Amérique centrale ou l'ami au Canada ne dispose pas des fonds nécessaires ou n'est pas un parent suffisamment proche. Les personnes dans cette dernière situation sont très inquiètes et désireuses de fournir toute l'aide possible à leurs parents et amis d'Amérique centrale.

Il y a aussi au Canada des gens qui sont disposés à aider les réfugiés d'Amérique centrale. II s'agit dans bien des cas de membres de la communauté chrétienne chez qui la persécution ouverte de l'Église en Amérique centrale a suscité un ardent désir de faire tout ce qui était possible. La plupart des sectes religieuses offrent déjà de l'aide par l'intermédiaire de leurs services d'urgence et de leurs programmes de développement, mais certaines paroisses se disent prêtes à recourir au programme de parrainage, pourvu qu'un mécanisme adéquat soit établi à cet égard.

\section{LA PROPOSITION}

Instituer un programme spécial modeste à l'intention des réfugiés d'Amerique centrale, de façon qu'il soit possible d'accueillir au moins cinq cents réfugiés du Guatemala et du Salvador, parrainés par le gouvernement, en sus des 1000 réfugiés latino-américains que le Canada prévoit acceillir en 1981.

Rendre admissible au programme tout réfugié ayant un contact quelconque au Canada. II pourrait s'agir d'un résident canadien apparenté au réfugié ou d'un ami disposé, à titre d'ami de la famille à aider le réfugié à s'établir au Canada.

Mettre en place un programme spécial modeste à l'intention des réfugiés d'Amérique centrale, pour qu'il soit possible d'accueillir au moins cinq cents réfugiés du Guatemala et du Salvador, parrainés par des groupes privés identifiés par des institutions religieuses d'Amérique centrale. 